\title{
THE TOPOLOGY AND DYNAMICS OF FLOWS
}

\author{
MICHAEL C. SULLIVAN
}

\begin{abstract}
After a brief survey of various types of flows (Morse-Smale, Smale, Anosov, \& partially hyperbolic) we focus on Smale flows on $S^{3}$. However, we do give some consideration to Smale flows on other three-manifolds and to Smale diffeomorphisms.
\end{abstract}

\section{FLOWS}

Let $M$ be a compact connected Riemannian manifold without boundary. Let $\|\cdot\|$ be the norm on the tangent bundle $T M$ and $d(\cdot, \cdot)$ the metric induced on $M$. By a flow on $M$ we mean a smooth function $f: M \times \mathbb{R} \rightarrow M$ such that $f(f(x, s), t)=f(x, s+t)$ and $f(x, 0)=x$. Much of what we describe in this sections for flows carries over with suitable modifications to diffeomorphisms.

The chain recurrent set of a flow $f$ is

$$
\begin{gathered}
\mathcal{R}=\left\{x \in M \mid \forall \epsilon>0, \exists\left\{x_{0}=x, x_{1}, x_{2}, \ldots, x_{k}\right\} \subset M \&\right. \\
\left\{t_{1}, t_{2}, \ldots, t_{k}\right\} \subset \mathbb{R}^{+} \text {such that } \\
\left.d\left(f\left(x_{i}, t_{i}\right), x_{i+1}\right)<\epsilon, i=1, \ldots, k-1, \text { and } d\left(f\left(x_{k}, t_{k}\right), x_{0}\right)<\epsilon\right\}
\end{gathered}
$$

The chain recurrent set of a flow is said to have a hyperbolic structure if the tangent bundle of the manifold structure can be written as a Whitney sum $T_{\mathcal{R}}=$ $E^{u} \oplus E^{c} \bigoplus E^{s}$ of sub-bundles invariant under $D f$ where $E_{x}^{c}$ is the subspace of $T M_{x}$ corresponding to the orbit of $x$ and such that there are constants $C>0$ and $\lambda>0$ for which $\left\|D f_{t}(v)\right\| \leq C e^{-\lambda t}\|x\|$ for $v \in E^{s}, t \geq 0$ and $\left\|D f_{t}(v)\right\| \geq 1 / C e^{\lambda t}\|x\|$ for $v \in E^{u}, t \geq 0$.

Steve Smale showed that when $\mathcal{R}$ is hyperbolic it is the closure of the periodic orbits of the flow. Smale also showed that when $\mathcal{R}$ is hyperbolic it has a finite decomposition into compact invariant sets called basic sets:

Theorem (Spectral Decomposition Theorem). Suppose that the chain recurrent set $\mathcal{R}$ of a flow has a hyperbolic structure. Then $\mathcal{R}$ is a finite disjoint union of compact invariant sets $\Lambda_{1}, \Lambda_{2}, \ldots, \Lambda_{k}$ where each $\Lambda_{i}$ contains an orbit that is dense in $\Lambda_{i}$.

We define respectively the stable and unstable manifolds of an orbit $\mathcal{O}$ in a flow $f$.

$$
\begin{gathered}
W^{s}(\mathcal{O})=\{y \in M \mid d(f(y, t), f(x, t)) \rightarrow 0 \text { as } t \rightarrow \infty \text { for some } x \in \mathcal{O}\} \\
W^{u}(\mathcal{O})=\{y \in M \mid d(f(y, t), f(x, t)) \rightarrow 0 \text { as } t \rightarrow-\infty \text { for some } x \in \mathcal{O}\}
\end{gathered}
$$

Date: April 21, 2006.

2000 Mathematics Subject Classification. 37D20, 37D05, 37D15, 37B25, 54H20, 37B10 . 
That these are manifolds is a classical result of Hirsch and Pugh [28] referred to as the Stable Manifold Theorem. A flow is structurally stable if it is topologically equivalent, i.e. there is a homeomorphism taking orbits to orbits preserving the flow direction, to flows obtained by small enough perturbations.

A flow with hyperbolic chain recurrent set $\mathcal{R}$ satisfies the transversality condition if the stable and unstable manifolds of $\mathcal{R}$ always meet transversally. A flow (or diffeomorphism) that has a hyperbolic chain recurrent set and satisfies the transversality condition is structurally stable; see [21, Theorem 1.10] for references. The converse - known as the $C^{1}$ Stability Conjecture - was proposed by Palis and Smale in [36] has been proven by $\mathrm{Hu}$ [29] for dimension 3 and for arbitrary dimension by Hayashi [27]; see also [50].

For the three dimensional case the basic sets of $C^{1}$ structurally stable flows may be of the following types: isolated fixed points; isolated closed orbits; suspensions of nontrivial irreducible $s$ hifts of finite type (SFTs) (see [30] for definitions of terms for symbolic dynamics) - these have infinitely many periodic orbits but rational zeta functions; two-dimensional attractors or repellers, e.g., a suspension of Pylkin's attractor - these are modeled by inverse limits of branched one-dimensional manifolds [51]; and lastly, if the invariant hyperbolic set is the whole of $M$, we have an Anosov flow.

If the chain recurrent set of a flow is hyperbolic, consists of a finite collection of periodic orbits and fixed points, and satisfies the transversality condition, we have a Morse-Smale flow. Daniel Asimov showed that for $n \neq 3$ all $n$-manifolds (possibly with boundary), subject to certain obvious Euler characteristic criteria, support nonsingular Morse-Smale flows [1]. (A nonsingular flow is just a flow without fixed points.) John Morgan has characterized which 3-manifolds (possibly with boundary) support nonsingular Morse-Smale flows [35] and Masaaki Wada has determined which links can be realized as the invariant set of a nonsingular Morse-Smale flow on $S^{3}$ [49]; see also [13]. Wada's result shows, for example, that the figure- 8 knots cannot be realized in a nonsingular Morse-Smale flow on $S^{3}$. Thus, the existence of a figure- 8 knot in a Morse-Smale flow on $S^{3}$ forces a fixed point. Bifurcations of nonsingular Morse-Smale flows on $S^{3}$ are studied in [12]. Given a link $L$ in some orientable 3-manifold, Masahico Saito [40] shows how to modify the 3-manifold (by forming connected sums with $S^{2} \times S^{1}$ pieces) so that the new 3-manifold has a nonsingular Morse-Smale flow with $L$ as part of its chain recurrent set (actually he shows a bit more than this).

If the chain recurrent set of a flow is at most one-dimensional and satisfies the transversality condition the flow is known as a Smale flow. Basic sets which are not isolated fix points or closed orbits are suspensions of SFTs and must be saddle sets. There are no chaotic attractors or repellers.

Anosov flows arose from the study of geodesic flows on surfaces. Thus, "unit tangent bundles of all surfaces with genus greater than one" support Anosov flows. And so too do "all manifolds that can be obtained by suspending Anosov diffeomorphisms of $T^{2}$." 1 There are no Anosov flows on $S^{3}$. It is known that a 3 -manifold for which every co-dimension one foliation has a Reeb component does not support

\footnotetext{
${ }^{1}$ Keith Burns, private communication.
} 
an Anosov flow. There are infinity many such manifolds [39]. In general, "[It is] not known at all which manifolds have Anosov flows." 2

Question. Which 3-manifolds support Anosov flows?

There has been a great deal of interest in partially hyperbolic flows; see [38]. These can have singular hyperbolic invariant sets in which a saddle fixed point cannot be isolated from an invariant set with infinitely many periodic orbits. Together they form a compact invariant attractor or repeller. The Lorenz attractor is a standard example. The first return map of a cross section may be conjugate to a shift space with a countably infinite alphabet. They are not structurally stable. See [33]. Morales and Pacifico have shown that generically a flow on a 3-manifold either has infinitely many sinks or sources, or (exclusive) has a chain recurrent set that is hyperbolic or singular hyperbolic. Their result implies that a generic flow on a 3-manifold has an attractor or a repeller. This is done in [34], a paper that should be widely read. They raise the following question in Conjecture 1.3.

Question. Can every $C^{1}$ vector field on a closed 3-manifold be approximated by a $\mathbf{1 0 0 2}$ ? vector field exhibiting a homoclinic tangency or by a singular Axiom A one without cycles? (See [34] for definitions and details.)

Their conjecture is "yes".

The rest of this chapter is devoted to Smale flows.

\section{Templates FOR BAsiC Sets}

Let $B$ be a basic set of a Smale flow that is the suspension of a nontrivial irreducible SFT. We can pick a neighborhood of $B$ that will be foliated by stable manifolds. If we form a quotient space by collapsing along the stable direction we derive a two dimensional branched manifold $\mathcal{T}_{B}$ known as a template. The original flow will induce a semi-flow on the template. A theorem of Joan Birman and Robert Williams asserts that there is a one-to-one correspondence between the periodic orbits of $B$ and $\mathcal{T}_{B}$ that preserves the knot type of each periodic orbit and how they are linked [4]; see also [24, Theorem 2.2.4]. Templates allow us to "see" basic sets. The simplest example is the Lorenz template shown in Figure 1 on the left. One can recover the basic set by taking an inverse limit of the template's semi-flow. For the Lorenz template the basic set is a suspension of the full 2-shift.

Templates, slightly modified, are used to model singular hyperbolic attractors, see Figure 1 on the right [3], and Plykin-like attractors - here the templates have no boundary and are harder to draw [24, Figure 3.15].

It is natural to ask which knots and links exist on a given template. (For basic definitions of knot theory see [11]). Let $L(m, n)$ denote the Lorenz-like template constructed from the Lorenz template by adding $m$ half twists in the left band and $n$ in the right band; by symmetry $L(m, n)=L(n, m)$. It is known that for $n \geq 0$ that the knots in $L(0, n)$ are prime positive braids [52], while for $n<0$ all knots and links are in $L(0, n)$ [23]. A template that contains all knots and links is called universal. A template is positive if it can be placed in a braid form with all

\footnotetext{
${ }^{2}$ Sergio Fenley, private communication.
} 

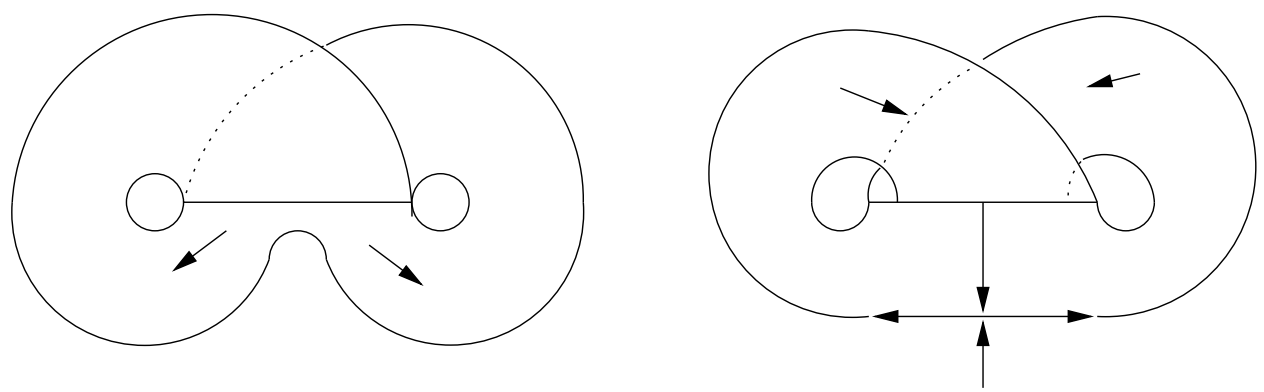

FiguRe 1. Left: Lorenz template for a saddle set. Right: Lorenz template for a singular hyperbolic Lorenz attractor.

crossings having the same orientation. For positive templates there is a bound on the number of prime factors of the supported knots [46]. For $m$ and $n$ positive the $L(m, n)$ knots have at most two prime factors, while for $L(-1,-1)$ the bound is three [47]. Even though $L(-1,-1)$ is not a positive template it can be presented so the all the crossing are positive, but not while it is in braid form [41]. When both $m$ and $n$ are negative it is known the $L(m, n)$ does not support all links [24, Proposition 3.2.21].

? 1003 Question. Is there a general way to characterize which templates are universal? Is there a bound on the number of prime factors of knots in templates that can be presented with only one crossing type?

When the "standard" suspension of the Plykin attractor is placed in a flow on $S^{3}$ it contains a copy of $L(0,-1)$ [24, Proposition 3.2.18] and thus contains all knots and links [24, Proposition 3.2.18]. Rob Ghrist found that the same was true for every Plykin-like attractor he studied, but no general theorem is known here.

? 1004 Question. Are there any attractors which are "standard" embeddings of Plykin-like attractors that do not have all knots and links?

\section{TWIST-WISE FLOW EQUIVALENCE}

Two flows are topologically equivalent if there is an orbit-wise homeomorphism between them that preserves the flow direction. Two SFTs are flow equivalent if their suspensions are topologically equivalent. Two non-negative square matrices are flow equivalent if they generate flow equivalent SFTs. In particular, incidence matrices of first return maps of any two cross sections to the same flow of this type are flow equivalent, although the two return maps need not be topologically conjugate (the usual equivalence relation for SFTs). Topologically conjugate SFTs are flow equivalent.

For nontrivial, irreducible non-negative square matrices, John Franks [19] has shown that flow equivalence is completely determined by two easy to compute 
invariants. They are the Parry-Sullivan number, denoted $P S$, and the BowenFranks group, denoted $B F$, derived in [37] and [9], respectively. If $M$ is any nonnegative integral $n \times n$ matrix then

$$
P S=\operatorname{det}(I-M) \quad \text { and } \quad B F=\frac{\mathbb{Z}^{n}}{(I-M) \mathbb{Z}^{n}} .
$$

We note that $|P S|$ is the order of $B F$ if $B F$ is finite, otherwise $P S=0$. (The trivial matrices are the permutation matrices. These generate SFTs and suspension flows with a only a finite number of orbits, all closed.)

Flow equivalence only looks at basic sets, not at the ambient flows they may be embedded in. For example, the inverse limit flows of $L(0,0)$ and $L(0,1)$ are topologically equivalent since both are suspensions of the full 2-shift. Yet they look different: one has a (orientation reversing) twisted band, the other does not. To capture this twist-wise flow equivalence was introduced in [43]. We add additional information to the incidence matrices by using a $t$ if the first return map is orientation reversing on a member of the Markov partition. This may require refining the Markov partition, which can always be done. Call these modified incidence matrices twist matrices. For $L(0,2 n)$ and $L(0,2 n+1)$ we get twist matrices

$$
\left[\begin{array}{ll}
1 & 1 \\
1 & 1
\end{array}\right] \quad \text { and } \quad\left[\begin{array}{ll}
1 & 1 \\
t & t
\end{array}\right]
$$

respectively. We take $t^{2}=1$ in all matrix calculations to mimic the fact the composition the first return map with itself is orientation preserving. Formally, twist matrices have entries of the form $a t+b$, with $a$ and $b$ nonnegative integers, and are just matrices over the semi-group ring $\mathbb{Z}^{+}[\mathbb{Z} / 2]$.

The topological interpretation is as follows. Take two basic sets of flows. Suppose they are flow equivalent. If we can extend the homeomorphism into the tangent bundles so that the stable and unstable sub-bundles are preserved, we say the embedded basic sets are twist-wise flow equivalent or sometimes ribbon equivalent; visually it is easier to extend the homeomorphism just a little, say $\epsilon>0$, into the tangent bundle. Then the extended homeomorphism will take annuli to annuli, Mobius bands to Mobius bands, and infinite strips to infinite strips. Two twist matrices are twist-wise flow equivalent if they correspond to ribbon equivalent embedded basic sets.

There are several easy to compute invariants. If $T(t)$ is a twist matrix $T( \pm 1)$ is defined by evaluating $T(t)$ at $t= \pm 1$. Let

$$
P S^{ \pm}=\operatorname{det}(I-T( \pm 1)) \quad \text { and } \quad B F^{ \pm}=\frac{\mathbb{Z}^{n}}{(I-T( \pm 1)) \mathbb{Z}^{n}} .
$$

Then $P S^{+}$and $B F^{+}$are clearly invariants since $T(1)$ is just the incidence matrix. It is shown in $[41,42]$ that $P S^{-}$and $B F^{-}$are also invariants that distinguish twist matrices not distinguished by $P S^{+}$and $B F^{+}$.

Next, let $A=\left[\begin{array}{ll}0 & 1 \\ 1 & 0\end{array}\right]$. Then regard $T(A)$ as the $2 n \times 2 n$ matrix obtained by replacing each $t$ with $A$ and each 1 with the $2 \times 2$ identity matrix: $a+b t \rightarrow\left[\begin{array}{ll}a & b \\ b & a\end{array}\right]$. 
Define

$$
P S^{\partial}=\operatorname{det}(I-T(A)) \quad \text { and } \quad B F^{\partial}=\frac{\mathbb{Z}^{2 n}}{(I-T(A)) \mathbb{Z}^{2 n}} .
$$

It is shown in [42] that $P S^{\partial}$ and $B F^{\partial}$ are invariants of twist-wise flow equivalence. While $P S^{\partial}=P S^{+} \times P S^{-}$there are examples of pairs of twist matrices which are not distinguished by $P S^{ \pm}$and $B F^{ \pm}$but are distinguished by $B F^{\partial}$.

Example. Let $A=\left[\begin{array}{cc}3 & 1+t \\ 1+t & 3\end{array}\right]$ and $B=\left[\begin{array}{cc}3 & 1+t \\ 2 & 3\end{array}\right]$. We get $P S^{+}=0$,

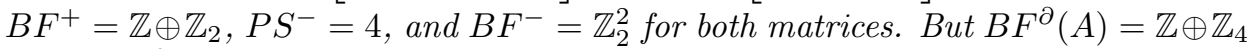
while $B F^{\partial}(B)=\mathbb{Z}_{2}^{2}$. Thus, $A$ and $B$ are in distinct twist-wise flow equivalence classes.

Example. For $\left[\begin{array}{ll}1 & 1 \\ 1 & 1\end{array}\right]$ and $\left[\begin{array}{ll}t & 1 \\ 1 & 1\end{array}\right]$ we get $P S^{ \pm}=-1$, which implies all three BF groups are trivial. Yet, the suspension flow of the first matrix has no Mobius bands while the suspension flow of the second clearly does.

Orientability, that is whether or not the ribbon set contains a Mobius band, is itself an invariant and easy to check for. If the twist matrix $T$ is $n \times n$ then it is enough to check the diagonal entries of the first $n$ powers of $T$. If no $t$ 's appear, then there are no Mobius bands in the ribbon set.

In [10] a complete algebraic invariant is produced. But, it is not known whether or not it is computable. An expository account is given in [45]. Given a matrix $A$ over $\mathbb{Z}^{+}[\mathbb{Z} / 2]$ let $(I-A)_{\infty}$ be the $\mathbb{N} \times \mathbb{N}$ matrix equal to $I-A$ in its upper left hand corner and the infinite identity everywhere else. The theorem below is Theorem 6.8 of [45] which is a special case of Theorem 6.3 of [10]; see either of these for the definition of essentially irreducible.

Theorem. Let $A$ and $B$ be nontrivial essentially irreducible matrices over $\mathbb{Z}^{+}[\mathbb{Z} / 2]$ and assume they are nonorientable. Then $A$ and $B$ are twist-wise flow equivalent if and only if there is an $S L\left(\mathbb{N}, \mathbb{Z}\left[\mathbb{Z}_{2}\right]\right)$ equivalence from $(I-A)_{\infty}$ to $(I-B)_{\infty}$.

Classifying matrices up to SL-equivalence over a PID is done by using an algorithm to convert them to a standard normal form (the Smith normal form). However, $\mathbb{Z}[\mathbb{Z} / 2]$ is not a PID: $(1+t)(1-t)=0$. It is unknown if an analogous algorithm exists for matrices over $\mathbb{Z}[\mathbb{Z} / 2]$ or if SL-equivalence is decidable here.

?1005 Question. Is there an algorithm to classify square matrices over $\mathbb{Z}[\mathbb{Z} / 2]$ up to SL-equivalence? This would then settle the problem of determining twist-wise flow equivalence of basic sets of Smale flows.

\section{Putting The PIECES TOGETher AND REALizATION PROBlems}

Now we look at how the basic sets can be pieced together to form Smale flows, with an emphasis on non-singular flows. This can be looked at from two prospectives. We will first review some results of John Franks that determine which basic sets can fit together to form a nonsingular flow on $S^{3}$ and some generalizations. Next we ask just how the basic sets can fit together. 
Suppose there is a single attracting closed orbit and a single repelling closed orbit in a nonsingular Smale flow on $S^{3}$. All other basic sets are saddle sets. Then we can compute the absolute value of the linking number of the attracting repelling pair as follows. Suppose there are $n$ saddle sets and that for the $i$-th one $\operatorname{det} T(t)$ is given by $a_{i}+t b_{i}$. Then the absolute value of the linking number is the product $\left|a_{1}-b_{1}\right| \cdots\left|a_{n}-b_{n}\right|[16]$. For example, the template $L(1,1)$ gives linking number 3.

The structure matrix of an embedded basic set is just its twist matrix evaluated at $t=-1$. In [18] the following are proved. If $S$ is any structure matrix of a basic set, then there exists a nonsingular Smale flow $\phi_{t}$ on some 3-manifold with a basic set $B$ whose structure matrix is $A$ and every other basic set of $\phi_{t}$ consists of a single attracting or repelling closed orbit (Theorem 1 ). If there exists a nonsingular Smale follow on $S^{3}$ with basic set $B$ with structure matrix $S$ then there exists a nonsingular Smale flow of $S^{3}$ with a twist-wise flow equivalent basic set with all other basic sets being attracting or repelling closed orbits (Proposition 3.2). Furthermore, if $\operatorname{det}(I-S) \neq 0$ then the group $\mathbb{Z}^{n} /(I-S) \mathbb{Z}^{n}$ is cyclic (Theorem 3.3). Thus,

$$
\left[\begin{array}{ll}
1 & 2 \\
2 & 1
\end{array}\right]
$$

cannot realized as the structure matrix of a basic set in a nonsingular Smale flow on $S^{3}$.

Question. Are there any other obstructions (besides [18, Theorem 3.3]) to the $\mathbf{1 0 0 6}$ ? realization of structure matrices in nonsingular Smale flows on $S^{3}$ ?

Finally, in [20] we have an abstract classification of nonsingular Smale flows on $S^{3}$. The major new tool is the Lyapunov graph. Given a Smale flow on a manifold there exist a smooth function from the manifold to the reals which is non-increasing with respect to the flow parameter. Thus, each basic set goes to a point. This is called a Lyapunov function. The Lyapunov graph is defined by identifying connected components of the inverse images of points in the real line. Each vertex of the graph is a point whose connected component contained a basic set. A vertex is labeled by the basic set it is associated with. Edges are oriented by the flow direction.

Suppose $\Gamma$ is an abstract Lyapunov graph whose sinks and sources are each labeled with a single attracting or repelling periodic orbit and suppose each remaining vertex is labeled with the suspension of a subshift of finite type. Then $\Gamma$ is associated with a nonsingular Smale flow on $S^{3}$, if and only if the following are satisfied. (1) The graph $\Gamma$ is a tree with one edge attached to each source and each sink vertex. (2) If $v$ is a saddle vertex whose basic set has incidence matrix $M$ and with $e_{v}^{+}$entering edges and $e_{v}^{-}$exiting edges then

$$
\begin{gathered}
e_{v}^{+} \leq \mathbb{Z}_{M}+1, \\
e_{v}^{-} \leq \mathbb{Z}_{M}+1, \\
\mathbb{Z}_{M}+1 \leq e_{v}^{+}+e_{v}^{-} .
\end{gathered}
$$


Here $\mathbb{Z}_{M}$ is a the Zeeman number defined by $\operatorname{dim} \operatorname{ker}\left(\left(I-M_{2}\right): \mathbb{Z}_{2}^{n} \rightarrow \mathbb{Z}_{2}^{n}\right)$, where $M_{2}$ is the mod 2 reduction of $M, \mathbb{Z}_{2}$ is the integers $\bmod 2$, and $n$ is the size of $M$. (Ketty de Rezende has generalized Lyapunov graphs to Smale flows with singularities [15].)

Thus, if there is a single attracting closed orbit and a single repelling closed orbit $\mathbb{Z}_{M}=0$ or 1 . The converse holds as well. Further, if $|a-b|=1$ we know that the linking number is 1 . But, we do not know whether or not they can or must form a Hopf link.

To see how the basic sets fit together involves mostly ad hoc cut-and-paste arguments. It is unlikely that a complete Wada like theorem will be found.

Smale flows on $S^{3}$ where there is a single attracting and a single repelling closed orbit, and a single saddle set modeled by an embedding of the Lorenz template were studied in [44]. It was show that the attractor/repeller pair either formed a Hopf link or a trefoil and meridian, and that the template was $L(0,2 n)$ for some $n$.

Let $\phi_{t}$ be a Smale flow on $M^{3}$. We say a template $T$ (we include the embedding in $M^{3}$ in the definition of the symbol $T$ ), is realized by $\phi$ is $\phi$ has a basic set modeled by a template isotopic to $T$ in $M^{3}$. In his Ph.D. dissertation [31], Vadim Meleshuk studies realization of templates by Smale flows on $S^{3}$. Without any other restriction, all templates are realizable with only fixed point basic sets [Theorem 3.3.1 $]^{3}$ A template can be realized in a flow whose only other basic sets are fixed point attractors and repeller if and only if certain easy to check topological criteria are meet [Theorem 3.3.2].

Meleshuk then switches his attention to nonsingular Smale flows (NSFs). He shows that every template is realizable by a nonsingular Smale flow on some 3manifold [Theorem 3.4.1]. On $S^{3}$, he gives a complete criteria for when a template can be realized by a NSF [Theorems 3.5.1, 3.5.6, 3.6.3]. In some cases a template $T$ is realizable with only attractors and repellers, but other times $T$ may force other saddle sets. For example, take a Lorenz template and tie a figure- 8 knot in one band. By [31] it can be realized in a nonsingular Smale flow, but by [44] it cannot be realized with just a single repeller and attractor as the only other basic sets. What other basic sets could be forced?

Following [22] Meleshuk works with thickened templates. These are handle bodies whose boundaries are partitioned into 2-dimensional exit and entrance sets, separated by loops (the tangent set). They retract naturally to the branched manifold version of templates. He explores, using homological machinery, relations between the entrance and exit sets. For example, he shows that if $T$ is realizable in a nonsingular Smale flow on $S^{3}$ and the entrance and exit sets are connected, then they are diffeomorphic [Theorem 3.10.6], and conjectures that if a template can be realizable in a nonsingular Smale flow on $S^{3}$ with only one attractor and one repeller, then the exit and entrance sets must be diffeomorphic [Conjecture 3.10.11].

One of the motivations for the study of Smale flows is their connection to Anosov flows. Any Anosov flow can be turned into a nonsingular Smale flow via two

\footnotetext{
${ }^{3}$ Meleshuk gives an independent proof, but the result can also be derived from a more general theorem of William Bloch [7].
} 
surgery moves $[4,24]$. Of course the underlying manifold will not be $S^{3}$. Yet, beyond [4] little has been done to exploit this. Ketty de Rezende along with several collaborators has developed the theory of Lyapunov graphs of Smale flows to other manifolds [14, 2]. Sue Goodman has characterized when a flow on an arbitrary 3-manifold with a one-dimensional hyperbolic set has a transverse foliation noting the importance of transverse foliations in the theory of Anosov flows; see [26] and also $[25,54]$. Are these tools sufficient to give a more unified understanding of nonsingular Smale flows on manifolds that support Anosov flows and Anosov flows?

\section{Bonatti's GeOMETRIC TYPE}

A Smale diffeomorphism is a hyperbolic map with zero dimensional basic sets. A Smale flow always has Smale diffeomorphisms as cross sections. In a series of paper's Bonatti et al $([5,8,6,48])$ have developed a new approach to the study of Smale flows on 3-manifolds and Smale diffeomorphisms on surfaces. The idea is to encode geometric information along with a Markov partition. This data includes twist data as in the twist matrices, but also includes "order" information; it is encoded as a geometrized Markov partition However, the geometrized Markov partition is not presented as a matrix but a mapping; whence it is not clear how to compute invariants from it.

We shall give an example from a paper by Vago [48]. A Smale diffeomorphism $f$ on a disk takes two large rectangles $r_{1}$ and $r_{2}$ to images shown in Figure 2. The horizontal strips, $h_{11}, h_{12}, h_{13}$ in $r_{1}$ and $h_{21}, h_{22}, h_{23}$ in $r_{2}$ are taken to the vertical strips $v_{24}, v_{23}, v_{22}, v_{21}, v_{11}, v_{12}$, respectively. From this one constructs the map $\phi$, from $(1,2) \times(1,2,3)$ (more typically the subset of realized indices) into $(1,2) \times(1,2,3,4) \times(+,-)$ given by

$$
\begin{aligned}
& \phi(1,1)=(2,4,-) \\
& \phi(1,2)=(2,3,+) \\
& \phi(1,3)=(2,2,-) \\
& \phi(2,1)=(1,4,-) \\
& \phi(2,2)=(1,3,+) \\
& \phi(2,3)=(1,2,-)
\end{aligned}
$$

where the signs tells us whether the orientation has been reversed or not.

Geometrized Markov partitions have been used to prove theorems giving necessary and sufficient conditions for the existence of congujacies. We shall state two, but shall not define all the terms, as we only intend to give the reader the flavor of this area.

Theorem ([8]; translated in [48]). Let $f$ and $g$ be two Smale diffeomorphisms on compact surfaces, and let $K$ and $L$ be hyperbolic saturated sets of $f$ and $g$ respectively, without attractors or repellers. Then $f$ and $g$ are conjugate on domains of $K$ and $L$ if and only if $(K, f)$ and $(L, g)$ admit Markov partitions of the same geometrical type.

Theorem ([5]). Let $X$ and $Y$ be Smale vectors fields on compact orientable 3manifolds. Let $K$ and $L$ be saturated saddle sets in $X$ and $Y$ respectively. Suppose that $K$ and $L$ admit good Markov partitions of the same geometrical type. Then 

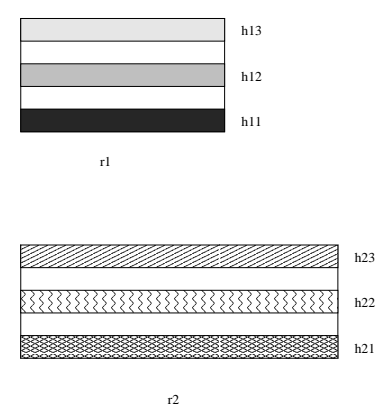

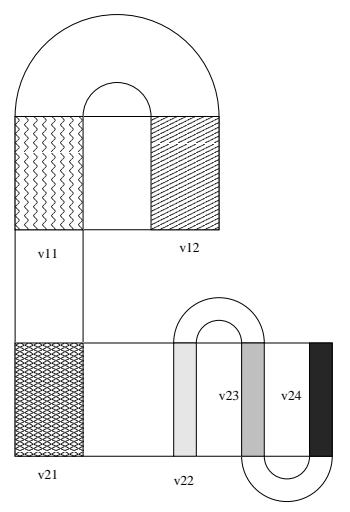

Figure 2. A Smale map

there exist invariants neighborhoods of $K$ and $L$ where the restrictions of the field $X$ and $Y$ respectively, are equivalent.

Order is nonabelian (obviously). What is needed is a nonabelian theory of symbolic dynamics. Bob Williams has developed a determinant for nonabelian matrices that contains some knot theoretic data for Lorenz attractors [53]. Could his matrices be modified to contain order data? They might capture part of the geometrized Markov partition in matrix form and thus facilitate the search for computable invariants. Another approach is to to use the skew-products systems in [10]. There the skew-products are of SFTs over finite groups. When the group is $\mathbb{Z} / 2$ we get twist-wise flow equivalence. But the results in [10] hold for all finite groups including nonabelian groups. I have tired to find a way to use permutations groups to capture some of the order information in the geometrized Markov partition, but without success. When the map is iterated the order information does not seem to behave is a "group-like" manner. Could some non-group algebraic structure be used? But then would skew-products be meaningful??

? 1007 Question. How can we get computable invariants that capture some of the order information in Bonatti's geometrized Markov partition?

\section{REFERENCES}

[1] Asimov, Daniel. Round handles and non-singular Morse-Smale flows. Ann. of Math. (2) 102 (1975), no. 1, 41-54. MR0380883 (52 \#1780)

[2] Bertolim, Maria Alice; Mello, Margarida Pinheiro \& de Rezende, Ketty Abaroa Dynamical and topological aspects of Lyapunov graphs. Qual. Theory Dyn. Syst. 4 (2003), no. 2, 181203 (2004). MR2129718 (2006b:37025)

[3] Birman, Joan \& Williams, R. F. Knotted periodic orbits in dynamical systems-I : Lorenz's equations. Topology, 22(1):47-82, 1983.

[4] Birman, Joan; Williams, R. F. Knotted periodic orbits in dynamical systems-II: knot holders for fibered knots. Cont. Math., 20:1-60, 1983.

[5] Béguin, F. \& Bonatti, C. Flots de Smale en dimension 3: présentations finies de voisinages invariants d'ensembles selles. (French) [Smale flows in dimension 3: finite presentations of 
invariant neighborhoods of saddle sets] Topology 41 (2002), no. 1, 119-162. MR1871244 (2002k:37058)

[6] F. Béguin, C. Bonatti and J. Vieitez. Construction de flots de Smale en dimension 3. (French. English, French summary) [Construction of three-dimensional Smale flows] Ann. Fac. Sci. Toulouse Math. (6) 8 (1999), no. 3, 369-410. MR1751171 (2002b:37037)

[7] Bloch, W. L. Extending flows from isolated invariant sets. Ergodic Theory Dynam. Systems 15 (1995), no. 6, 1031-1043. MR1366306 (96m:58127)

[8] Bonatti, C. \& Langevin, R. Difféomorphismes de Smale des surfaces. (French) [Smale diffeomorphisms of surfaces] With the collaboration of E. Jeandenans. Astérisque No. 250 (1998), viii+235 pp. MR1650926 (99m:58123)

[9] R. Bowen and J. Franks. Homology of zero dimensional basic sets. Annals of Mathematics, 106:73-92, 1972.

[10] Boyle, M. \& Sullivan, M. Equivariant flow equivalence for shifts of finite type. Proceedings of the London Mathematical Society, Vol 91, Part 1, July 2005.

[11] Burde, G. \& Zieschang, H. Knots: de Gruyter Studies in Mathematics, 5. Second edition, Walter de Gruyter \& Co., Berlin, 2003. MR1959408 (2003m:57005)

[12] B. Campos, J. Martinez Alfaro, P. Vindel. Bifurcations of links of periodic orbits in non-singular Morse-Smale systems on $S^{3}$, Nonlinearity 10 (1997) 1339-1355. MR1473388 (98i:58133)

[13] Casasayas, J., Martinez Alfaro, J., and Nunes, A. Knots and links in integrable Hamiltonian systems. J. Knot Theory Ramifications 7 (1998), no. 2, 123-153.

[14] Cruz, R. N. \& de Rezende, K. A. Cycle rank of Lyapunov graphs and the genera of manifolds. Proc. Amer. Math. Soc. 126 (1998), no. 12, 3715-3720. MR1618654 (99f:58112)

[15] de Rezende, Ketty. Smale flows on the three-sphere. Trans. Amer. Math. Soc. 303 (1987), no. 1, 283-310. MR0896023 (88k:58125)

[16] Franks, John. Non-singular flows on $S^{3}$ with hyperbolic chain-recurrent set, Rocky Mountain J. Math., Vol. 7, No. 3, Summer 1977, 539-546. MR0488164 (58 \#7728)

[17] Franks, John. Knots, links and symbolic dynamics. Ann. of Math. 116 (1981), 279-294. MR0621015 (83h:58074)

[18] Franks, John Symbolic dynamics in flows on three-manifolds., Trans. Amer. Math. Soc. 279 (1983), 231-236. MR0704612 (84h:58112)

[19] Franks, John. Flow equivalence of subshifts of finite type. Ergodic Theory and Dynamical Systems, Vol. 4 (1984) No. 1, 53-66. MR0758893 (86j:58078)

[20] Franks, John Nonsingular Smale flows on $S^{3}$, Topology, 24 (1985), 265-282. MR0815480 (87d:58110)

[21] Homology and dynamical systems. CBMS Regional Conference Series in Mathematics, 49. Published for the Conference Board of the Mathematical Sciences, Washington, D.C.; by the American Mathematical Society, Providence, R. I., 1982. viii+120 pp. Reprinted, with corrections, 1989. MR0669378 (84f:58067)

[22] J. Franks and M. Sullivan. Flows with knotted closed orbits. Handbook of geometric topology, 471-497, North-Holland, Amsterdam, 2002. MR1886676 (2003c:37023)

[23] R. W. Ghrist. Branched two-manifolds supporting all links. Topology 36 (1997), no. 2, 423448. MR1415597 (98b:57009)

[24] R. W. Ghrist, P. J. Holmes and M. C. Sullivan. Knots and Links in Three-Dimensional Flows: Lecture Notes in Mathematics, 1654, Springer-Verlag, 1997. MR1480169 (98i:58199)

[25] Goodman, Sue. Vector fields with transverse foliations. Topology 24 (1985), no. 3, 333-340. MR0815483 (87k:57022a)

[26] Goodman, Sue. Vector fields with transverse foliations. II. Ergodic Theory Dynam. Systems 6 (1986), no. 2, 193-203. MR0857197 (87k:57022b)

[27] Hayashi, Shuhei. Connecting invariant manifolds and the solution of the $C^{1}$ stability and $\Omega$-stability conjectures for flows. Ann. of Math. (2) 145 (1997), no. 1, 81-137. Errata: Ann. of Math. (2) 150 (1999), no. 1, 353-356. MR1432037 (98b:58096) 
[28] Hirsch, Morris W.\& Pugh, Charles C. Stable manifolds and hyperbolic sets. 1970 Global Analysis (Proc. Sympos. Pure Math., Vol. XIV, Berkeley, Calif., 1968) pp. 133-163, Amer. Math. Soc., Providence, R.I.

[29] Hu, Sen. A proof of $C^{1}$ stability conjecture for three-dimensional flows. Trans. Amer. Math. Soc. 342 (1994), no. 2, 753-772.

[30] D. Lind and B. Marcus. An Introduction to Symbolic Dynamics and Coding, Cambridge University Press, Cambridge 1995. MR1369092 (97a:58050)

[31] V. Meleshuk, "Embedding Templates in Flows," Ph.D. dissertation, Northwestern University, 2002.

[32] Morales, C. A. 3-manifolds supporting Anosov flows and Abelian normal subgroups. Topology Appl. 143 (2004), no. 1-3, 249-255. MR2081015 (2005d:57032)

[33] Morales, C. A., Pacifico, M. J. \& Pujals, E. R. Singular hyperbolic systems. Proc. Amer. Math. Soc. 127 (1999), no. 11, 3393-3401. MR1610761 (2000c:37034)

[34] Morales, C. A. \& Pacifico, M. J. A dichotomy for three-dimensional vector fields. Ergodic Theory Dynam. Systems 23 (2003), no. 5, 1575-1600. MR2018613 (2005a:37030)

[35] Morgan, John W. Nonsingular Morse-Smale flows on 3-dimensional manifolds. Topology 18 (1979), no. 1, 41-53. MR0528235 (80j:58043)

[36] Palis, J. \& Smale, S. Structural stability theorems. 1970 Global Analysis (Proc. Sympos. Pure Math., Vol. XIV, Berkeley, Calif., 1968) pp. 223-231, Amer. Math. Soc., Providence, R.I. MR0267603 (42 \#2505)

[37] Parry, William \& Sullivan, Dennis. A topological invariant of flows on 1-dimensional spaces. Topology, 14 (1975), no. 4, 297-299. MR0405385 (53 \#9179)

[38] Pesin, Yakov B. Lectures on partial hyperbolicity and stable ergodicity. Zurich Lectures in Advanced Mathematics. European Mathematical Society (EMS), Zrich, 2004. vi+122 pp. MR2068774 (2005j:37039)

[39] Roberts, R., Shareshian, J.\& Stein, M. Infinitely many hyperbolic 3-manifolds which contain no Reebless foliation. J. Amer. Math. Soc. 16 (2003), no. 3, 639-679. MR1969207 (2004e:57023)

[40] Saito, Masahico. On closed orbits of Morse-Smale flows on 3-manifolds. Bull. London Math. Soc. 23 (1991), no. 5, 482-486. MR1141020 (94a:58107)

[41] Sullivan, M. Knots in Robinson's attractor are positive. J. Knot Thy. and Ram., 7 (1998), no. $1,115-121$.

[42] Sullivan, M. Invariants of twist-wise flow equivalence. Discrete Contin. Dynam. Systems, 4 (1998), no. 3, 475-484. MR1612752 (99c:58137)

[43] Sullivan, M. An invariant of basic sets of Smale flows, Ergod. Th. E Dynam. Sys., 17 (1997), no. 6, 1437-1448. Errata: 18 (1998), no. 4, 1047 MR1488328 (99f:58157a)

[44] Sullivan, M. Visually Building Smale flows in $S^{3}$. Topology and Its Applications, 106 (2000), no. 1, 1-19. MR1769328 (2001g:37025)

[45] Twistwise flow equivalence and beyond .... With an appendix by Mike Boyle and the author. Contemp. Math., 385, Algebraic and topological dynamics, 171-186, Amer. Math. Soc., Providence, RI, 2005. MR2180236

[46] Sullivan, M. Knots on a positive template have a bounded number of prime factors. Algebraic and Geometric Topology, Volume 5 (2005) 536-576. MR2153116 (2006c:57007)

[47] Sulliva, M. Factoring Families of Positive Knots on Lorenz-like Templates. Preprint 2005.

[48] G. M. Vago. Conjugate unstable manifolds and their underlying geometrised Markov partitions. Proceedings of the French-Japanese Conference "Hyperspace Topologies and Applications" (La Bussire, 1997). Topology Appl. 104 (2000), no. 1-3, 255-291. MR1780989 (2002b:37036)

[49] Wada, Masaaki. Closed orbits of nonsingular Morse-Smale flows on $S^{3}$. J. Math. Soc. Japan 41 (1989), no. 3, 405-413. MR0999505 (90g:58059)

[50] Wen, Lan. On the $C^{1}$ stability conjecture for flows. J. Differential Equations 129 (1996), no. 2, 334-357. MR1404387 (97j:58082)

[51] Williams, R. F. Expanding attractors. Inst. Hautes tudes Sci. Publ. Math. No. 43 (1974), 169-203. MR0348794 (50 \#1289) 
[52] Williams, R. F. Lorenz Knots are Prime. Ergod. Th. E Dynam. Sys. 4 (1983) 147-163. MR0758900 (86c:58103)

[53] Williams, R. F. A new zeta function, natural for links. From Topology to Computation: Proceedings of the Smalefest (Berkeley, CA, 1990), 270-278, Springer, New York, 1993 MR1246126 (94k:58116)

[54] Yano, Koichi. Nonsingular Morse-Smale flows on 3-manifolds which admit transverse foliations. Foliations (Tokyo, 1983), 341-358, Adv. Stud. Pure Math., 5, North-Holland, Amsterdam, 1985. MR0877338 (88f:57051) 